\title{
UNIQUENESS PROBLEM WITH TRUNCATED MULTIPLICITIES IN VALUE DISTRIBUTION THEORY
}

\author{
HIROTAKA FUJIMOTO
}

\begin{abstract}
In 1929, H. Cartan declared that there are at most two meromorphic functions on $\mathbb{C}$ which share four values without multiplicities, which is incorrect but affirmative if they share four values counted with multiplicities truncated by two. In this paper, we generalize such a restricted H. Cartan's declaration to the case of maps into $P^{N}(\mathbb{C})$. We show that there are at most two nondegenerate meromorphic maps of $\mathbb{C}^{n}$ into $P^{N}(\mathbb{C})$ which share $3 N+1$ hyperplanes in general position counted with multiplicities truncated by two. We also give some degeneracy theorems of meromorphic maps into $P^{N}(\mathbb{C})$ and discuss some other related subjects.
\end{abstract}

\section{$\S 1$. Introduction}

In 1926, R. Nevanlinna showed that, for two distinct nonconstant meromorphic functions $f$ and $g$ on the complex plane $\mathbb{C}$, they cannot have the same inverse images for five distinct values, and $g$ is a special type of linear fractional transformation of $f$ if they have the same inverse images counted with multiplicities for four distinct values([10]). In [2] [4], the author gave several types of generalizations of these theorems to the case of meromorphic maps of $\mathbb{C}^{n}$ into $P^{N}(\mathbb{C})$. He considered two distinct meromorphic maps $f$ and $g$ satisfying the condition that $\nu\left(f, H_{j}\right)=\nu\left(g, H_{j}\right)$ for $q$ hyperplanes $H_{1}, H_{2}, \ldots, H_{q}$ in $P^{N}(\mathbb{C})$ located in general position, where $\nu\left(f, H_{j}\right)$ means the map of $\mathbb{C}^{n}$ into $\mathbb{Z}$ whose value $\nu\left(f, H_{j}\right)(a)\left(a \in \mathbb{C}^{n}\right)$ is the intersection multiplicity of the images of $f$ and $H_{j}$ at $f(a)$. He proved that $q \leq 3 N+1$ if either $f$ or $g$ is (linearly) nondegenerate, and $q \leq 2 N+2$ if either $f$ or $g$ is algebraically nondegenerate.

It is reasonable to ask whether these results remain valid regardless of multiplicity or not. There are several results without multiplicities in some restricted situations. To state some of them, we take a nondegenerate meromorphic map $g$ of $\mathbb{C}^{n}$ into $P^{N}(\mathbb{C})$, a positive integer $\ell_{0}$ and $q$ hyperplanes

Received June 26, 1997. 
$H_{1}, H_{2}, \ldots, H_{q}$ in $P^{N}(\mathbb{C})$ located in general position with

$$
\operatorname{dim} g^{-1}\left(H_{i} \cap H_{j}\right) \leq n-2 \quad(1 \leq i<j \leq q)
$$

and consider the set $\mathcal{F}\left(H_{1}, \ldots, H_{q} ; g ; \ell_{0}\right)$ of all nondegenerate meromorphic maps $f: \mathbb{C}^{n} \rightarrow P^{N}(\mathbb{C})$ satisfying the conditions;

(a) $\min \left(\nu\left(f, H_{j}\right), \ell_{0}\right)=\min \left(\nu\left(g, H_{j}\right), \ell_{0}\right)$ for $1 \leq j \leq q$,

(b) $f=g$ on $\bigcup_{j=1}^{q} g^{-1}\left(H_{j}\right)$.

In [12], L. Smiley gave the following uniqueness theorem.

THEOREM 1.1. ([12]) If $q \geq 3 N+2$, then $f=g$ for any map $f \in$ $\mathcal{F}\left(H_{1}, \ldots, H_{q} ; g ; 1\right)$.

In 1929, as an improvement of the above-mentioned Nevanlinna's results, $\mathrm{H}$. Cartan declared that there are at most two meromorphic functions on $\mathbb{C}$ which have the same inverse images regardless of multiplicities for four distinct values $([1])$. However, in [13], N. Steinmetz gave examples which show that H. Cartan's declaration is false. In relation to this, S. Ji obtained the following result by the use of H. Cartan's original idea.

THEOREM 1.2. ([9]) Assume that $q=3 N+1$. Then, for arbitrary three maps $f^{0}, f^{1}, f^{2} \in \mathcal{F}\left(H_{1}, \ldots, H_{q} ; g ; 1\right)$, the map $F:=\left(f^{0}, f^{1}, f^{2}\right): \mathbb{C}^{n} \rightarrow$ $P^{N}(\mathbb{C}) \times P^{N}(\mathbb{C}) \times P^{N}(\mathbb{C})$ is algebraically degenerate, namely, the image $F\left(\mathbb{C}^{n}\right)$ is included in a (special) proper subvariety of $P^{N}(\mathbb{C}) \times P^{N}(\mathbb{C}) \times$ $P^{N}(\mathbb{C})$.

We can easily show that $\mathrm{H}$. Cartan's declaration is affirmative if we assume that meromorphic functions on $\mathbb{C}$ share distinct four values counted with multiplicities truncated by 2 . The main purpose of this paper is to generalize such a restricted H. Cartan's declaration to the case of meromorphic maps into $P^{N}(\mathbb{C})$.

Our main results are stated as follows:

THEOREM 1.3. Suppose that $q \geq 2 N+2$ and take arbitrary $N+2$ maps $f^{0}, \ldots, f^{N+1}$ in $\mathcal{F}\left(H_{1}, \ldots, H_{q} ; g ; N(N+1) / 2+N\right)$. Then, there are $N+1$ hyperplanes $H_{j_{0}}, H_{j_{1}}, \ldots, H_{j_{N}}$ among $H_{j}^{\prime}$ s such that, if we choose homogeneous coordinates $\left(w_{0}: \cdots: w_{N}\right)$ on $P^{N}(\mathbb{C})$ with $H_{j_{i}}=\left\{w_{i}=0\right\}$ 
$(0 \leq i \leq N)$ and take representations $f^{k}=\left(f_{0}^{k}: \cdots: f_{N}^{k}\right)$ with nonzero holomorphic functions $f_{j}^{k}$, then

$$
\frac{f_{i}^{1}}{f_{j}^{1}}-\frac{f_{i}^{0}}{f_{j}^{0}}, \frac{f_{i}^{2}}{f_{j}^{2}}-\frac{f_{i}^{0}}{f_{j}^{0}}, \ldots, \frac{f_{i}^{N+1}}{f_{j}^{N+1}}-\frac{f_{i}^{0}}{f_{j}^{0}}
$$

are linearly dependent over $\mathbb{C}$ for each pair $(i, j)$ with $0 \leq i, j \leq N$.

THEOREM 1.4. If $q=3 N+1$, then $\mathcal{F}\left(H_{1}, \ldots, H_{q} ; g ; 2\right)$ contains at most two maps.

There are several open problems related to the above results. It is desirable to be shown that the condition (b) for the definition of $\mathcal{F}\left(H_{1}, \ldots, H_{q} ; g\right.$; $\left.\ell_{0}\right)$ is omitted or replaced by a weaker one and that the number $q=3 N+1$ in Theorem 1.4 is replaced by a smaller one. As related matters to uniqueness problem in value distribution theory for meromorphic maps into $P^{N}(\mathbb{C})$, there are some similar results for the Gauss map of complete minimal surfaces in $\mathbb{R}^{m}$ (cf., [6], [7] and [8]). It is an interesting problem to get more precise results for the Gauss map of a complete minimal surface which are analogous to Theorems 1.3 and 1.4 .

In [5], the author introduced the notion of $\kappa$-nondegeneracy for meromorphic maps into $P^{N}(\mathbb{C})$ (cf., Definition 2.8). We prove Theorems 1.3 and 1.4 in more precise forms for $\kappa$-nondegenerate meromorphic maps in $\S 5$ (cf., Theorem 5.1). We give also a degeneracy theorem of meromorphic maps into $P^{N}(\mathbb{C})$ in $\S 4$ (cf., Theorem 4.9).

\section{$\S 2$. Preliminaries from Nevanlinna theory}

Let $F$ be a nonzero holomorphic function on a domain $D$ in $\mathbb{C}^{n}$. For a set $\alpha=\left(\alpha_{1}, \ldots, \alpha_{n}\right)$ of nonnegative integers, we set $|\alpha|:=\alpha_{1}+\cdots+\alpha_{n}$ and $\mathcal{D}^{\alpha} F:=\frac{\partial^{|\alpha|} F}{\partial^{\alpha_{1}} z_{1} \cdots \partial^{\alpha_{n}} z_{n}}$. We define the map $\nu_{F}^{0}: D \rightarrow \mathbb{Z}$ by

$$
\nu_{F}^{0}(z):=\max \left\{m ; \mathcal{D}^{\alpha} F(z)=0 \text { for all } \alpha \text { with }|\alpha|<m\right\} \quad(z \in D) .
$$

We mean by a divisor on a domain $D$ in $\mathbb{C}^{n}$ a map $\nu$ of $D$ into $\mathbb{Z}$ such that, for each $a \in D$, there are nonzero holomorphic functions $F$ and $G$ on a connected neighborhood $U(\subset D)$ of $a$ such that $\nu(z)=\nu_{F}^{0}(z)-\nu_{G}^{0}(z)$ for each $z \in U$ outside an analytic set of dimension $\leq n-2$. Two divisors are regarded as the same if they are identical outside an analytic set of 
dimension $\leq n-2$. For a divisor $\nu$ on $D$ we set $|\nu|:=\overline{\{z: \nu(z) \neq 0\}}$, which is a purely $(n-1)$-dimensional analytic subset of $D$ or empty.

Take a nonzero meromorphic function $\varphi$ on a domain $D$ in $\mathbb{C}^{n}$. For each $a \in D$, we choose nonzero holomorphic functions $F$ and $G$ on a neighborhood $U(\subset D)$ of $a$ such that $\varphi=F / G$ on $U$ and $\operatorname{dim} F^{-1}(0) \cap G^{-1}(0) \leq$ $n-2$, and we define the divisors $\nu_{\varphi}^{\alpha}$ by $\nu_{\varphi}^{\alpha}:=\nu_{F-\alpha G}^{0}$ for $\alpha \in \mathbb{C}$ and by $\nu_{\varphi}^{\alpha}:=\nu_{G}$ for $\alpha=\infty$, which are independent of choices of $F$ and $G$ and so globally well-defined on $D$.

Let $f$ be a meromorphic map of $\mathbb{C}^{n}$ into $P^{N}(\mathbb{C})$, which is nondegenerate, namely, whose image $f\left(\mathbb{C}^{n}\right)$ is not included in any hyperplane in $P^{N}(\mathbb{C})$. We take holomorphic functions $f_{0}, f_{1}, \ldots, f_{N}$ such that $I_{f}:=\left\{z \in \mathbb{C}^{n} ; f_{0}(z)=\right.$ $\left.\cdots=f_{N}(z)=0\right\}$ is of dimension at most $n-2$ and $f(z)=\left(f_{0}(z): \cdots\right.$ : $\left.f_{N}(z)\right)$ on $\mathbb{C}^{n}-I_{f}$ in terms of homogeneous coordinates on $P^{N}(\mathbb{C})$. We call such a representation $f=\left(f_{0}: \cdots: f_{N}\right)$ a reduced representation of $f$.

For $z=\left(z_{1}, \ldots, z_{n}\right) \in \mathbb{C}^{n}$ we set $\|z\|:=\left(\sum_{j=1}^{n}\left|z_{j}\right|^{2}\right)^{1 / 2}$ and define

$$
\begin{array}{rlrl}
B(r) & :=\left\{z \in \mathbb{C}^{n} ;\|z\|<r\right\}, & S(r) & :=\left\{z \in \mathbb{C}^{n} ;\|z\|=r\right\}, \\
d^{c} & :=\frac{\sqrt{-1}}{4 \pi}(\bar{\partial}-\partial), & v & :=\left(d d^{c}\|z\|^{2}\right)^{n-1}, \\
\sigma & :=d^{c} \log \|z\|^{2} \wedge\left(d d^{c} \log \|z\|^{2}\right)^{n-1} .
\end{array}
$$

Set $\|f\|:=\left(\left|f_{0}\right|^{2}+\cdots+\left|f_{N}\right|^{2}\right)^{1 / 2}$. The order function of $f$ is given by

$$
T(r, f)=\int_{S(r)} \log \|f\| \sigma-\int_{S(1)} \log \|f\| \sigma .
$$

For a divisor $\nu$ on $\mathbb{C}^{n}$, we define the counting function of $\nu$ by

$$
N(r, \nu):=\int_{1}^{r} \frac{n(t)}{t^{2 n-1}} d t \quad(1<r<+\infty)
$$

where $n(t):=\int_{|\nu| \cap B(t)} \nu v$ for $n \geq 2$ and $n(t):=\sum_{|z| \leq t} \nu(z)$ for $n=1$.

Consider a hyperplane

$$
H: a_{0} w_{0}+\cdots+a_{N} w_{N}=0
$$

in $P^{N}(\mathbb{C})$, where $A:=\left(a_{0}, \ldots, a_{N}\right) \neq(0, \ldots, 0)$. We set $F_{H}:=a_{0} f_{0}+$ $\cdots+a_{N} f_{N}$ and define the divisor $\nu(f, H)$ by $\nu(f, H)(z):=\nu_{F_{H}}^{0}(z)\left(z \in \mathbb{C}^{n}\right)$ which is rephrased as the intersection multiplicity of the image of $f$ and $H$ 
at $f(z)$. For a positive integer $m$ or $m=+\infty$ we define the (truncated) counting function of $H$ for $f$ by

$$
N_{m}(r, H) \equiv N_{m}^{f}(r, H):=N(r, \min (\nu(f, H), m)) .
$$

For brevity, we set $N(r, H):=N_{+\infty}^{f}(r, H)$. Setting

$$
\psi_{f}(H)(z):=\frac{\|f\|\|A\|}{\left|F_{H}\right|}
$$

we define the proximity function of $H$ by

$$
m_{f}(r, H):=\int_{S(r)} \log \left|\psi_{f}(H)\right| \sigma-\int_{S(1)} \log \left|\psi_{f}(H)\right| \sigma .
$$

We now state the first main theorem in Nevanlinna theory.

THEOREM 2.1. $T(r, f)=N(r, H)+m_{f}(r, H)$ for all hyperplanes $H$.

We may identify $\overline{\boldsymbol{C}}:=\boldsymbol{C} \cup\{\infty\}$ with $P^{1}(\mathbb{C})$ and, in another situation, with the set $P^{1}(\mathbb{C})^{*}$ of all hyperplanes in $P^{1}(\mathbb{C})$. For a nonconstant meromorphic function $\varphi$ on $\mathbb{C}$ and every $\alpha \in \bar{C}, m_{\varphi}(r, \alpha)$ means the proximity function of $\alpha \in P^{1}(\mathbb{C})^{*}$ for $\varphi: C^{n} \rightarrow P^{1}(\mathbb{C})$. On the other hand, the proximity function of a meromorphic function $\varphi$ is classically defined by

$$
m(r ; \varphi):=\int_{S(r)} \log ^{+}|\varphi| \sigma
$$

where $\log ^{+} x:=\max (\log x, 0)$ for $x \geq 0$. As is easily seen, we have

(2.2) $m(r ; \varphi)=m_{\varphi}(r, \infty)+O(1)$.

(2.3) For nonzero meromorphic functions $\varphi_{1}, \varphi_{2}$ with $\varphi_{1}+\varphi_{2} \not \equiv 0$, we set $\psi_{1}:=\varphi_{1} \varphi_{2}$ and $\psi_{2}:=\varphi_{1}+\varphi_{2}$. Then,

$$
\begin{aligned}
m\left(r ; \psi_{k}\right) & \leq m\left(r ; \varphi_{1}\right)+m\left(r ; \varphi_{2}\right)+O(1) \quad(k=1,2), \\
T\left(r, \psi_{k}\right) & \leq T\left(r, \varphi_{1}\right)+T\left(r, \varphi_{2}\right)+O(1) \quad(k=1,2) .
\end{aligned}
$$

Now, take two distinct hyperplanes

$$
H_{j}: a_{j 0} w_{0}+a_{j 1} w_{1}+\cdots+a_{j N} w_{N}=0 \quad(j=1,2)
$$

and consider a meromorphic function

$$
F_{f}^{H_{1}, H_{2}}:=\frac{a_{10} f_{0}+\cdots+a_{1 N} f_{N}}{a_{20} f_{0}+\cdots+a_{2 N} f_{N}} .
$$

We can easily prove 


$$
T\left(r, F_{f}^{H_{1}, H_{2}}\right) \leq T(r, f)+O(1)
$$

As usual, by the notation " $\| P$ " we mean the assertion $P$ holds for all $r \in[0,+\infty)$ excluding a Borel subset $E$ of the interval $[0,+\infty)$ with $\int_{E} d r<+\infty$. The following so-called logarithmic derivative lemma acts essential roles in Nevanlinna theory.

TheOREM 2.6. For any $\alpha=\left(\alpha_{1}, \ldots, \alpha_{n}\right)$, we have

$$
\| m\left(r ; \frac{D^{\alpha}\left(F_{f}^{H_{1}, H_{2}}\right)}{F_{f}^{H_{1}, H_{2}}}\right)=o(T(r, f)) .
$$

For the proof, refer to [5] and [11, Lemma 3.11].

Consider a vector-valued meromorphic function $F=\left(f_{0}, \ldots, f_{N}\right)$. For each $a \in \mathbb{C}^{n}$, we denote by $\mathcal{M}_{a}$ the set of all germs of meromorphic functions at $a$ and, for $\kappa=1,2, \ldots$, by $\mathcal{F}^{\kappa}$ the $\mathcal{M}_{a}$-submodule of $\mathcal{M}_{a}^{N+1}$ which is generated by the set $\left\{D^{\alpha} F:=\left(D^{\alpha} f_{0}, \cdots, D^{\alpha} f_{N}\right) ;|\alpha| \leq \kappa\right\}$. Set $\ell_{F}(\kappa):=$ $\operatorname{rank}_{\mathcal{M}_{a}} \mathcal{F}^{\kappa}$, which does not depend on each $a \in \mathbb{C}^{n}$. For a meromorphic $\operatorname{map} f=\left(f_{0}: f_{1}: \cdots: f_{N}\right): \mathbb{C}^{n} \rightarrow P^{N}(\mathbb{C})$, we set $\ell_{f}(\kappa):=\ell_{\left(f_{0}, \cdots, f_{N}\right)}(\kappa)$.

By [5, Proposition 4.3], we have the following:

(2.7) A meromorphic map $f: \mathbb{C}^{n} \rightarrow P^{N}(\mathbb{C})$ is nondegenerate if and only if there is some $\kappa$ such that $\ell_{f}(\kappa)=N+1$.

Definition 2.8. A nondegenerate meromorphic map $f: \mathbb{C}^{n} \rightarrow P^{N}(\mathbb{C})$ is said to be $\kappa$-nondegenerate if $\ell_{f}(\kappa)=N+1$.

We can show the following:

Proposition 2.9. Every nondegenerate meromorphic map of $\mathbb{C}^{n}$ into $P^{N}(\mathbb{C})$ of rank $k(\leq \min (N, n))$ is $(N-k+1)$-nondegenerate.

Proof. By the assumption, we have $\ell_{f}(1)=k+1$. Set $\kappa_{0}:=\min \{\kappa$; $\left.\ell_{f}(\kappa)=N+1\right\}$. Since $\ell_{f}(\kappa-1)<\ell_{f}(\kappa)$ for each $\kappa\left(\leq \kappa_{0}\right)$ by [5, Lemma 4.6], we see

$$
N+1=\ell_{f}\left(\kappa_{0}\right)=\sum_{\kappa=2}^{\kappa_{0}}\left(\ell_{f}(\kappa)-\ell_{f}(\kappa-1)\right)+\ell_{f}(1) \geq\left(\kappa_{0}-1\right)+(k+1) .
$$

This gives $\kappa_{0} \leq N-k+1$. 
Definition 2.10. Assume that meromorphic functions $f_{0}, \ldots, f_{N}$ are linearly independent over $\mathbb{C}$. For $N+1$ vectors $\alpha^{i}:=\left(\alpha_{i 1}, \ldots, \alpha_{i n}\right)(0 \leq i \leq$ $N)$ composed of nonnegative integers $\alpha_{i j}$, we call a set $\alpha=\left(\alpha^{0}, \alpha^{1}, \ldots, \alpha^{N}\right)$ an admissible set for $F=\left(f_{0}, \ldots, f_{N}\right)$ if $\left\{\mathcal{D}^{\alpha^{0}} F, \ldots, \mathcal{D}^{\alpha^{\ell} F^{(\kappa)}} F\right\}$ is a basis of $\mathcal{F}^{\kappa}$ for each $\kappa=1,2, \ldots, \kappa_{0}:=\min \left\{\kappa^{\prime} ; \ell_{F}\left(\kappa^{\prime}\right)=N+1\right\}$.

By definition, for an admissible set $\left(\alpha^{0}, \alpha^{1}, \ldots, \alpha^{N}\right)$ we have

$$
\operatorname{det}\left(\mathcal{D}^{\alpha^{0}} F, \ldots, \mathcal{D}^{\alpha^{N}} F\right) \not \equiv 0
$$

As was shown in [5], we have the following:

Proposition 2.11. ([5, Proposition 4.5]) For arbitrarily given linearly independent meromorphic functions $f_{0}, \ldots, f_{N}$ on $\mathbb{C}^{n}$, there exists an admissible set $\alpha=\left(\alpha^{0}, \ldots, \alpha^{N}\right)$ with $|\alpha| \leq N(N+1) / 2$.

Proposition 2.12. ([5, Proposition 4.9]) Let $\alpha=\left(\alpha^{0}, \ldots, \alpha^{N}\right)$ be an admissible set for $F=\left(f_{0}, \ldots, f_{N}\right)$ and let $h$ be a holomorphic function. Then,

$$
\operatorname{det}\left(\mathcal{D}^{\alpha^{0}}(h F), \ldots, \mathcal{D}^{\alpha^{N}}(h F)\right)=h^{N+1} \operatorname{det}\left(\mathcal{D}^{\alpha^{0}} F, \ldots, \mathcal{D}^{\alpha^{N}} F\right) .
$$
$P^{N}(\mathbb{C})$.

We now state the second main theorem for meromorphic maps into

THEOREM 2.13. Let $f: \mathbb{C}^{n} \rightarrow P^{N}(\mathbb{C})$ be a $\kappa$-nondegenerate meromorphic map of $\mathbb{C}^{n}$ into $P^{N}(\mathbb{C})$ and $H_{1}, \ldots, H_{q}$ hyperplanes in general position. Then,

$$
\|(q-N-1) T(r, f) \leq \sum_{j=1}^{q} N_{\kappa}^{f}\left(r, H_{j}\right)+o(T(r, f)) .
$$

For the proof, refer to [5, Proposition 6.2].

\section{$\S 3$. Cartan's auxiliary functions}

Let $F_{0}, F_{1}, \ldots, F_{M}$ be nonzero meromorphic functions on $\mathbb{C}^{n}$, where $M \geq 1$. Take a set $\alpha:=\left(\alpha^{0}, \alpha^{1}, \ldots, \alpha^{M-1}\right)$ whose components $\alpha^{k}$ are composed of $n$ nonnegative integers, and set $|\alpha|=\left|\alpha^{0}\right|+\left|\alpha^{1}\right|+\cdots+\left|\alpha^{M-1}\right|$. 
Definition 3.1. We define Cartan's auxiliary function by $\Phi^{\alpha} \equiv \Phi^{\alpha}\left(F_{0}, \ldots, F_{M}\right)$

$$
:=F_{0} \cdots F_{M}\left|\begin{array}{cccc}
1 & 1 & \cdots & 1 \\
\mathcal{D}^{\alpha^{0}}\left(1 / F_{0}\right) & \mathcal{D}^{\alpha^{0}}\left(1 / F_{1}\right) & \cdots & \mathcal{D}^{\alpha^{0}}\left(1 / F_{M}\right) \\
\mathcal{D}^{\alpha^{1}}\left(1 / F_{0}\right) & \mathcal{D}^{\alpha^{1}}\left(1 / F_{1}\right) & \cdots & \mathcal{D}^{\alpha^{1}}\left(1 / F_{M}\right) \\
\mathcal{D}^{\alpha^{M-1}}\left(1 / F_{0}\right) & \mathcal{D}^{\alpha^{M-1}}\left(1 / F_{1}\right) & \cdots & \mathcal{D}^{\alpha^{M-1}}\left(1 / F_{M}\right)
\end{array}\right| .
$$

For the particular case $n=1, M=2$ and $\alpha=((0),(1))$, Cartan's auxiliary function is given by

$$
\Phi^{\alpha}(F, G, H)=F\left(\frac{G^{\prime}}{G}-\frac{H^{\prime}}{H}\right)+G\left(\frac{H^{\prime}}{H}-\frac{F^{\prime}}{F}\right)+H\left(\frac{F^{\prime}}{F}-\frac{G^{\prime}}{G}\right),
$$

which was introduced by $\mathrm{H}$. Cartan in [1].

Since we can rewrite $\Phi^{\alpha}$ as

$$
\Phi^{\alpha}=F_{0} F_{1} \cdots F_{M} \operatorname{det}\left(\mathcal{D}^{\alpha^{i}}\left(\frac{1}{F_{\ell}}-\frac{1}{F_{0}}\right) ; \begin{array}{l}
i=0,1, \ldots, M-1 \\
\ell=1,2, \ldots, M
\end{array}\right),
$$

we easily have the following proposition in view of Proposition 2.11.

Proposition 3.3. If $\Phi^{\alpha}\left(F_{0}, \ldots, F_{M}\right) \equiv 0$ for all $\alpha$ with $|\alpha| \leq M(M-$ $1) / 2$, then $1 / F_{1}-1 / F_{0}, \ldots, 1 / F_{M}-1 / F_{0}$ are linearly dependent over $\boldsymbol{C}$.

For the particular case $M=2$, we have the following:

Proposition 3.4. If $\Phi^{\alpha}(F, G, H)=0$ and $\Phi^{\alpha}(1 / F, 1 / G, 1 / H)=0$ for all $\alpha$ with $|\alpha| \leq 1$, then one of the following assertions holds;

(i) $F=G, G=H$ or $H=F$.

(ii) $F / G, G / H$ and $H / F$ are all constants.

Proof. Assume that the assertion (i) does not hold. By Proposition 3.3, we can find some nonzero vectors $(\lambda, \mu, \nu)$ and $\left(\lambda^{\prime}, \mu^{\prime}, \nu^{\prime}\right)$ with $\lambda+\mu+\nu=$ $\lambda^{\prime}+\mu^{\prime}+\nu^{\prime}=0$ such that

$$
\frac{\lambda}{F}+\frac{\mu}{G}+\frac{\nu}{H}=\lambda^{\prime} F+\mu^{\prime} G+\nu^{\prime} H=0 .
$$

Then, $\lambda \neq 0$ and $\lambda^{\prime} \neq 0$, because otherwise we have $G=H$. By the same reason, $\mu \mu^{\prime} \nu \nu^{\prime} \neq 0$. Here, we may assume $\lambda=\lambda^{\prime}=1$. Then, we get

$$
\mu^{\prime} \nu G^{2}+\left(\nu^{\prime} \nu+\mu^{\prime} \mu-1\right) G H+\nu^{\prime} \mu H^{2}=0 .
$$


This concludes that $G / H$ is a constant, and $F / G$ and $H / G$ are also constants.

Proposition 3.5. Assume that $1 / F_{1}-1 / F_{0}, \ldots, 1 / F_{M}-1 / F_{0}$ are linearly independent over $C$ and take an admissible set $\alpha=\left(\alpha^{0}, \ldots, \alpha^{M-1}\right)$ for them. If

$$
\nu^{\left[\ell_{0}\right]}:=\min \left(\nu_{F_{0}}^{0}, \ell_{0}\right)=\min \left(\nu_{F_{1}}^{0}, \ell_{0}\right)=\cdots=\min \left(\nu_{F_{M}}^{0}, \ell_{0}\right)
$$

for some $\ell_{0} \geq|\alpha|$, then $\nu_{\Phi^{\alpha}}^{0}\left(z_{0}\right) \geq \min \left(\nu^{\left[\ell_{0}\right]}\left(z_{0}\right), \ell_{0}-|\alpha|\right)$ for every $z_{0} \in$ $F_{0}^{-1}(0)$.

Proof. By the assumption, $F_{0}^{-1}(0)=\cdots=F_{M}^{-1}(0)$, which we denote by $A$. Since the set of all singularities of $A$ is an analytic set of dimension at most $n-2$, we may assume that $z_{0}$ is a regular point of $A$. We choose a nonzero holomorphic function $h$ on a neighborhood $U$ of $z_{0}$ such that $d h$ has no zero and $A \cap U=\{z \in U ; h(z)=0\}$. Set $m_{k}:=\nu_{F_{k}}^{0}\left(z_{0}\right)$ and $\varphi_{k}:=1 / F_{k}$ for $0 \leq k \leq M$. We can write $\varphi_{k}=h^{-m_{k}} \tilde{\varphi}_{k}$ on a neighborhood $V(\subset U)$ of $z_{0}$, where $\tilde{\varphi}_{k}$ are nowhere vanishing holomorphic functions on $V$.

We first consider the case $\nu^{\left[\ell_{0}\right]}\left(z_{0}\right)=\ell_{0}$. We rewrite $\Phi^{\alpha}$ as

$$
\Phi^{\alpha}=\sum_{i=0}^{M}(-1)^{i} F_{i} \psi_{i}
$$

with meromorphic functions

$$
\psi_{i}:=\operatorname{det}\left(\frac{\mathcal{D}^{\alpha^{\ell}} \varphi_{k}}{\varphi_{k}} ; \begin{array}{l}
k=0, \ldots, i-1, i+1, \ldots, M \\
\ell=0,1, \ldots, M-1
\end{array}\right)
$$

Here, as is easily seen by the induction on $\left|\alpha^{\ell}\right|$, we can write each $\mathcal{D}^{\alpha^{\ell}} \varphi_{k} / \varphi_{k}$ as

$$
\frac{\mathcal{D}^{\alpha^{\ell}} \varphi_{k}}{\varphi_{k}}=\frac{\psi_{k, \ell}}{h^{\left|\alpha^{\ell}\right|}}
$$

with a holomorphic function $\psi_{k, \ell}$, and each $\psi_{i}$ as

$$
\psi_{i}=\sum_{\ell=\left(\ell_{1}, \ldots, \ell_{M}\right)} \varepsilon(\ell) \frac{\mathcal{D}^{\alpha^{\ell_{1}}} \varphi_{0}}{\varphi_{0}} \cdots \frac{\mathcal{D}^{\alpha^{\ell_{i}}} \varphi_{i-1}}{\varphi_{i-1}} \frac{\mathcal{D}^{\alpha^{\ell_{i+1}}} \varphi_{i+1}}{\varphi_{i+1}} \cdots \frac{\mathcal{D}^{\alpha^{\ell_{M}}} \varphi_{M}}{\varphi_{M}}
$$

where $\ell=\left(\ell_{1}, \ldots, \ell_{M}\right)$ runs through all permutations of $0, \ldots, M-1$ and $\varepsilon(\ell)$ denotes the signature of a permutation $\ell$. These imply $\nu_{\psi_{i}}^{\infty} \leq|\alpha|$. 
Therefore, by the assumption $\nu_{F_{i}}^{0}\left(z_{0}\right) \geq \nu^{\left[\ell_{0}\right]}\left(z_{0}\right)=\ell_{0}$ we have $\nu_{\Phi^{\alpha}}\left(z_{0}\right) \geq$ $\ell_{0}-|\alpha|$.

We now assume that $(1 \leq) \nu^{\left[\ell_{0}\right]}\left(z_{0}\right)<\ell_{0}$. Then, by the assumption,

$$
m^{*}:=m_{0}=m_{1}=\cdots=m_{M}=\nu^{\left[\ell_{0}\right]}\left(z_{0}\right) \text {. }
$$

In this case, we write

$$
\Phi^{\alpha}=\frac{1}{\varphi_{0} \varphi_{1} \cdots \varphi_{M}} \operatorname{det}\left(D^{\alpha^{\ell}}\left(\varphi_{k}-\varphi_{0}\right) ; \begin{array}{l}
k=1, \ldots, M \\
\ell=0,1, \ldots, M-1
\end{array}\right),
$$

and $\varphi_{k}-\varphi_{0}=h^{-m^{*}}\left(\tilde{\varphi}_{k}-\tilde{\varphi}_{0}\right)$ with holomorphic functions $\tilde{\varphi}_{k}-\tilde{\varphi}_{0}$. We now apply Proposition 2.12 to show

$$
\begin{aligned}
\Phi^{\alpha} & =\frac{h^{m^{*}(M+1)}}{\tilde{\varphi}_{0} \cdots \tilde{\varphi}_{M}} \frac{1}{h^{m^{*} M}} \operatorname{det}\left(\mathcal{D}^{\alpha^{\ell}}\left(\tilde{\varphi}_{k}-\tilde{\varphi}_{0}\right) ; \begin{array}{c}
k=1,2, \ldots, M \\
\ell=0,1, \ldots, M-1
\end{array}\right) \\
& =\frac{h^{m^{*}}}{\tilde{\varphi}_{0} \cdots \tilde{\varphi}_{M}} \operatorname{det}\left(\mathcal{D}^{\alpha^{\ell}}\left(\tilde{\varphi}_{k}-\tilde{\varphi}_{0}\right) ; \begin{array}{c}
k=1,2, \ldots, M \\
\ell=0,1, \ldots, M-1
\end{array}\right) .
\end{aligned}
$$

These conclude $\nu_{\Phi^{\alpha}}^{0}\left(z_{0}\right) \geq m^{*}$. The proof of Proposition 3.5 is completed.

Proposition 3.7. Let $F_{0}, F_{1}, \ldots, F_{M}$ and $\alpha$ satisfy the same assumption as in Proposition 3.5. If $F_{0}=\cdots=F_{M} \not \equiv 0, \infty$ on a purely $(n-1)$-dimensional analytic subset $A$ of $\mathbb{C}^{n}$, then $\nu_{\Phi^{\alpha}}^{0}\left(z_{0}\right) \geq M$ for every $z_{0} \in A$.

Proof. By the same reason as in the proof of Proposition 3.5, we have only to show that $\nu_{\Phi^{\alpha}}\left(z_{0}\right) \geq M$ for all regular points $z_{0}$ of $A$ with $F_{k}\left(z_{0}\right) \neq 0$, $\infty(0 \leq k \leq M)$. Taking a holomorphic function $h$ on a neighborhood $U$ of $z_{0}$ such that $d h$ has no zero and $A \cap U=\{z \in U ; h(z)=0\}$, we write $\psi_{k}:=1 / F_{k}-1 / F_{0}=h \tilde{\psi}_{k}(1 \leq k \leq M)$ with nonzero holomorphic functions $\tilde{\psi}_{k}$ on a neighborhood of $z_{0}$. Then, by Proposition 2.12 we have

$$
\begin{aligned}
\Phi^{\alpha} & =F_{0} F_{1} \cdots F_{M} \operatorname{det}\left(\mathcal{D}^{\alpha^{\ell}} \psi_{k} ; \begin{array}{l}
\ell=0,1, \ldots, M-1 \\
k=1,2, \ldots, M
\end{array}\right) \\
& =F_{0} F_{1} \cdots F_{M} h^{M} \operatorname{det}\left(\mathcal{D}^{\alpha^{\ell}} \tilde{\psi}_{k} ; \begin{array}{l}
\ell=0,1, \ldots, M-1 \\
k=1,2, \ldots, M
\end{array}\right) .
\end{aligned}
$$

Thus, we obtain $\nu_{\Phi^{\alpha}}^{0}\left(z_{0}\right) \geq M$. 


\section{$\S 4$. Generalizations of Nevanlinna's results}

Let $g$ be a $\kappa$-nondegenerate meromorphic map of $\mathbb{C}^{n}$ into $P^{N}(\mathbb{C})$ and consider hyperplanes

$$
H_{j}: a_{j 0} w_{0}+\cdots+a_{j N} w_{N}=0 \quad(1 \leq j \leq q)
$$

in $P^{N}(\mathbb{C})$ which are located in general position and satisfy the condition

$$
\operatorname{dim} g^{-1}\left(H_{i} \cap H_{j}\right) \leq n-2 \quad(1 \leq i<j \leq q)
$$

For a given positive integer $\ell_{0}$, we denote by $\mathcal{F}_{\kappa}\left(H_{1}, \ldots, H_{q} ; g ; \ell_{0}\right)$ the set of all $\kappa$-nondegenerate meromorphic maps $f$ satisfying the conditions;

(4.2) $\min \left(\nu\left(f, H_{j}\right), \ell_{0}\right)=\min \left(\nu\left(g, H_{j}\right), \ell_{0}\right)$ for $1 \leq j \leq q$ and

(4.3) $f=g$ on $\bigcup_{j=1}^{q} g^{-1}\left(H_{j}\right)$.

We note that $\mathcal{F}_{N}\left(H_{1}, \ldots, H_{q} ; g ; \ell_{0}\right)$ coincides with $\mathcal{F}\left(H_{1}, \ldots, H_{q} ; g ; \ell_{0}\right)$ defined in $\S 1$ because of Proposition 2.9.

Take $M+1$ maps $f^{0}, f^{1}, \ldots, f^{M} \in \mathcal{F}_{\kappa}\left(H_{1}, \ldots, H_{q} ; g ; \ell_{0}\right)$ with reduced representations $f^{k}:=\left(f_{0}^{k}: \cdots: f_{N}^{k}\right)$ and set $T(r):=\sum_{k=0}^{M} T\left(r, f^{k}\right)$. With each $c=\left(c_{0}, \ldots, c_{N}\right) \in\left(\mathbb{C}^{N+1}\right)_{*}:=\mathbb{C}^{N+1}-\{0\}$ associating a hyperplane

$$
H_{c}:=\left\{\left(w_{0}: \cdots: w_{N}\right) ; c_{0} w_{0}+\cdots+c_{N} w_{N}=0\right\},
$$

we define

$\mathcal{C}:=\left\{c \in\left(\mathbb{C}^{N+1}\right)_{*} ; \operatorname{dim}\left(f^{k}\right)^{-1}\left(H_{c} \cap H_{j}\right) \leq n-2\right.$ for $\left.1 \leq j \leq q, 0 \leq k \leq M\right\}$.

Lemma 4.4. The set $\mathcal{C}$ is dense in $\left(C^{N+1}\right)_{*}$.

For the proof, refer to [9, Lemma 5.1].

Now, taking an arbitrary $c \in \mathcal{C}$, we define

$$
\begin{aligned}
F_{c}^{k j} & :=\frac{a_{j 0} f_{0}^{k}+\cdots+a_{j N} f_{N}^{k}}{c_{0} f_{0}^{k}+\cdots+c_{N} f_{N}^{k}}, \\
\psi_{c}^{k j} & :=\frac{1}{F_{c}^{k j}}-\frac{1}{F_{c}^{0 j}} \quad(1 \leq k \leq M, 1 \leq j \leq q),
\end{aligned}
$$

and assume that $\psi_{c}^{1 j_{0}}, \ldots, \psi_{c}^{M j_{0}}$ are linearly independent for some $j_{0}$ and $c \in \mathcal{C}$. 
Proposition 4.6. Taking an admissible set $\alpha=\left(\alpha_{0}, \ldots, \alpha_{M}\right)$ for the functions $\psi_{c}^{1 j_{0}}, \ldots, \psi_{c}^{M j_{0}}$, we consider $\Phi^{\alpha}:=\Phi^{\alpha}\left(F_{c}^{0 j_{0}}, \ldots, F_{c}^{M j_{0}}\right)(\not \equiv 0)$ and assume that $\ell_{0} \geq|\alpha|$. Then, for $0 \leq k \leq M$,

$$
\| N_{\ell_{0}-|\alpha|}^{f^{k}}\left(r, H_{j_{0}}\right)+M \sum_{j \neq j_{0}} N_{1}^{f^{k}}\left(r, H_{j}\right) \leq N\left(r, \nu_{\Phi^{\alpha}}^{0}\right) \leq T(r)+o(T(r)) .
$$

Proof. The first inequality is a direct result of Propositions 3.5 and 3.7. To show the second inequality, we apply Theorem 2.1 and (2.2), which give

$$
N\left(r, \nu_{\Phi^{\alpha}}^{0}\right) \leq T\left(r, \Phi^{\alpha}\right)+O(1)=N\left(r, \nu_{\Phi^{\alpha}}^{\infty}\right)+m\left(r ; \Phi^{\alpha}\right)+O(1)
$$

We easily see that a pole of $\Phi^{\alpha}$ is a zero or a pole of some $F_{c}^{k j_{0}}$, and $\Phi^{\alpha}$ is holomorphic at all zeros of $F_{c}^{k j_{0}}$ because of Proposition 3.5. These show that

$$
N\left(r, \nu_{\Phi^{\alpha}}^{\infty}\right) \leq \sum_{k=0}^{M} N\left(r, \nu_{F_{c}^{k j_{0}}}^{\infty}\right)
$$

On the other hand, using (2.3) and the representation (3.6), we have

$$
m\left(r ; \Phi^{\alpha}\right) \leq \sum_{k=0}^{M} m\left(r ; F_{c}^{k j_{0}}\right)+O\left(\sum_{0 \leq k \leq M, 0 \leq \ell \leq M-1} m\left(r ; \frac{\mathcal{D}^{\alpha^{\ell}} \varphi_{c}^{k j_{0}}}{\varphi_{c}^{k j_{0}}}\right)\right)+O(1)
$$

where $\varphi_{c}^{k j_{0}}=1 / F_{c}^{k j_{0}}$. By virtue of Theorems 2.1, 2.6 and (2.5), we conclude

$$
\begin{aligned}
\| N\left(r, \nu_{\Phi^{\alpha}}^{0}\right) & \leq \sum_{k=0}^{M}\left(N\left(r, \nu_{F^{k j_{0}}}^{\infty}\right)+m\left(r ; F_{c}^{k j_{0}}\right)\right)+o(T(r)) \\
& \leq \sum_{k=0}^{M} T\left(r, F_{c}^{k j_{0}}\right)+o(T(r)) \leq T(r)+o(T(r)) .
\end{aligned}
$$

In the above situation, we can prove the following:

Proposition 4.7. (i) For arbitrary $M \geq 1$ and $\ell_{0} \geq|\alpha|$,

$$
\|(q-N-1) T\left(r, f^{k}\right) \leq \frac{\kappa}{M} T(r)+N_{\kappa}^{f^{k}}\left(r, H_{j_{0}}\right)+o(T(r)) .
$$


(ii) For arbitrary $M \geq 1$ and $\ell_{0} \geq|\alpha|+1$,

$$
\begin{aligned}
\|(q-N-1) T\left(r, f^{k}\right) & \leq \kappa \sum_{j=1}^{q} N_{1}^{f^{k}}\left(r, H_{j}\right)+o\left(T\left(r, f^{k}\right)\right) \\
& \leq \frac{\kappa}{M} T(r)+\kappa\left(1-\frac{1}{M}\right) N_{1}^{f^{k}}\left(r, H_{j_{0}}\right)+o(T(r)) .
\end{aligned}
$$

(iii) If $M \geq \kappa$ and $\ell_{0} \geq|\alpha|+\kappa$, then

$$
\begin{aligned}
\|(q-N-1) T\left(r, f^{k}\right) & \leq \sum_{j=1}^{q} N_{\kappa}^{f^{k}}\left(r, H_{j}\right)+o\left(T\left(r, f^{k}\right)\right) \\
& \leq \frac{\kappa}{M} T(r)+\left(1-\frac{\kappa}{M}\right) N_{\kappa}^{f^{k}}\left(r, H_{j_{0}}\right)+o(T(r)) .
\end{aligned}
$$

Proof. For $M \geq 1$ and $\ell_{0} \geq|\alpha|$, Theorem 2.13 and Proposition 4.6 yield

$$
\begin{aligned}
\|(q-N-1) T\left(r, f^{k}\right) & \leq \kappa \sum_{j \neq j_{0}} N_{1}^{f^{k}}\left(r, H_{j}\right)+N_{\kappa}^{f^{k}}\left(r, H_{j_{0}}\right)+o\left(T\left(r, f^{k}\right)\right) \\
& \leq \frac{\kappa}{M} T(r)+N_{\kappa}^{f^{k}}\left(r, H_{j_{0}}\right)+o(T(r))
\end{aligned}
$$

which gives (i).

Assume that $\ell_{0} \geq|\alpha|+1$. In this case, we have

$$
\begin{aligned}
\|(q-N-1) T\left(r, f^{k}\right) \leq & \kappa \sum_{j=1}^{q} N_{1}^{f^{k}}\left(r, H_{j}\right)+o\left(T\left(r, f^{k}\right)\right) \\
= & \frac{\kappa}{M}\left(N_{1}^{f^{k}}\left(r, H_{j_{0}}\right)+M \sum_{j \neq j_{0}} N_{1}^{f^{k}}\left(r, H_{j}\right)\right) \\
& +\kappa\left(1-\frac{1}{M}\right) N_{1}^{f^{k}}\left(r, H_{j_{0}}\right)+o\left(T\left(r, f^{k}\right)\right) \\
\leq & \frac{\kappa}{M} T(r)+\kappa\left(1-\frac{1}{M}\right) N_{1}^{f^{k}}\left(r, H_{j_{0}}\right)+o(T(r)),
\end{aligned}
$$

which gives (ii).

Under the assumption $M \geq \kappa$ and $\tilde{\ell}_{0}:=\ell_{0}-|\alpha| \geq \kappa$, we have

$$
\|(q-N-1) T\left(r, f^{k}\right) \leq \sum_{j=1}^{q} N_{\kappa}^{f^{k}}\left(r, H_{j}\right)+o\left(T\left(r, f^{k}\right)\right)
$$




$$
\begin{aligned}
\leq & \frac{\kappa}{M}\left(N_{\tilde{\ell}_{0}}^{f^{k}}\left(r, H_{j_{0}}\right)+M \sum_{j \neq j_{0}} N_{1}^{f^{k}}\left(r, H_{j}\right)\right) \\
& +\left(N_{\kappa}^{f^{k}}\left(r, H_{j_{0}}\right)-\frac{\kappa}{M} N_{\tilde{\ell}_{0}}^{f^{k}}\left(r, H_{j_{0}}\right)\right)+o\left(T\left(r, f^{k}\right)\right) \\
\leq & \frac{\kappa}{M} T(r)+\left(1-\frac{\kappa}{M}\right) N_{\kappa}^{f^{k}}\left(r, H_{j_{0}}\right)+o(T(r)),
\end{aligned}
$$

which gives (iii). The proof of Proposition 4.7 is completed.

As a consequence of Proposition 4.7, we can prove the following improvement of Theorem 1.1.

Corollary 4.8. If $q \geq N+2 \kappa+2$, then $f=g$ for any map $f \in$ $\mathcal{F}_{\kappa}\left(H_{1}, \ldots, H_{q} ; g ; 1\right)$.

Proof. Assume that there is some $f \in \mathcal{F}_{\kappa}\left(H_{1}, \ldots, H_{q} ; g ; 1\right)$ with $f \neq g$ and set $f^{0}:=f$ and $f^{1}=g$. Then, for the functions $F_{c}^{k j}(k=0,1$, $j=0, \ldots, N)$ defined by (4.5), Cartan's auxiliary function is given by $\Phi^{0}\left(F_{c}^{0 j}, F_{c}^{1 j}\right)=F_{c}^{0 j}-F_{c}^{1 j}$. By the assumption, there is some $j_{0}$ such that $\Phi^{0}\left(F_{c}^{0 j_{0}}, F_{c}^{1 j_{0}}\right) \not \equiv 0$. Setting $M=1$ and $\ell_{0}=1$, we add the inequalities of Proposition 4.7 (ii) for the case $k=0,1$ to see

$$
\|(q-N-1) T(r) \leq 2 \kappa T(r)+o(T(r)) .
$$

Divide both sides by $T(r)$. We then obtain $q-N-1 \leq 2 \kappa$ as $r$ tends to $+\infty$, which contradicts the assumption.

We can also prove the following degeneracy theorem.

THEOREM 4.9. Take maps $f^{0}, f^{1}, \ldots, f^{\kappa+1} \in \mathcal{F}_{\kappa}\left(H_{1}, \ldots, H_{q} ; g ; \ell_{0}\right)$ for $\ell_{0}=\kappa(\kappa+1) / 2$. If $q>N+\kappa+2$, then

$$
\frac{\sum_{i=0}^{N} c_{i} f_{i}^{0}}{\sum_{i=0}^{N} a_{j i} f_{i}^{0}}, \frac{\sum_{i=0}^{N} c_{i} f_{i}^{1}}{\sum_{i=0}^{N} a_{j i} f_{i}^{1}}, \ldots, \frac{\sum_{i=0}^{N} c_{i} f_{i}^{\kappa+1}}{\sum_{i=0}^{N} a_{j i} f_{i}^{\kappa+1}}
$$

are linearly dependent over $C$ for each $j=1, \ldots, q$ and $c=\left(c_{0}, \ldots, c_{N}\right) \in \mathcal{C}$.

Proof. Assume that the conclusion is not valid. Then, there are some $j_{0}$ and $c \in \mathcal{C}$ such that $\Phi^{\alpha}\left(F_{c}^{0 j_{0}}, \ldots, F_{c}^{\kappa+1 j_{0}}\right) \not \equiv 0$, where $\alpha$ is an admissible 
set with $|\alpha| \leq \kappa(\kappa+1) / 2$ for $\left(\psi_{c}^{1 j_{0}}, \ldots, \psi_{c}^{\kappa+1 j_{0}}\right)$. We apply Proposition 4.7

(i) for the case $M:=\kappa+1$ to see

$$
\begin{aligned}
\|(q-N-1) T(r) & \leq(\kappa+2) \frac{\kappa}{\kappa+1} T(r)+\sum_{k=0}^{\kappa+1} N_{\kappa}^{f^{k}}\left(r, H_{j_{0}}\right)+o(T(r)) \\
& \leq\left(\frac{(\kappa+2) \kappa}{\kappa+1}+1\right) T(r)+o(T(r)) .
\end{aligned}
$$

In the same manner as in the proof of Corollary 4.8, we can conclude $q \leq$ $N+2+\kappa+\kappa /(\kappa+1)$. Since $q$ is an integer, we get $q \leq N+2+\kappa$. Thus, we have Corollary 4.9 .

Now, we give a generalization of Nevanlinna's results ([10, Satz 3].

THEOREM 4.10. Let $f^{0}, \cdots, f^{M} \in \mathcal{F}_{\kappa}\left(H_{1}, \cdots, H_{q} ; g ; \ell_{0}\right)$. Assume that $M \geq \kappa, \ell_{0} \geq M(M-1) / 2+\kappa$ and $\psi_{c}^{1 j_{0}}, \ldots, \psi_{c}^{M j_{0}}$ are linearly independent for some $j_{0}$ and $c \in \mathcal{C}$. Then, $q \leq N+\kappa+2$.

Moreover, if $q=N+\kappa+2$, then there is a set $E(\subset[0,+\infty))$ with $\int_{E} d r<+\infty$ such that

$$
\begin{gathered}
\lim _{r \rightarrow \infty, r \notin E} \frac{\sum_{j=1}^{q} N_{\kappa}^{f^{k}}\left(r, H_{j}\right)}{T\left(r, f^{k}\right)}=\kappa+1 \quad(0 \leq k \leq M), \\
\lim _{r \rightarrow \infty, r \notin E} \frac{T\left(r, f^{k}\right)}{T\left(r, f^{\ell}\right)}=1 \quad(0 \leq k<\ell \leq M)
\end{gathered}
$$

and, under the additional condition $M>\kappa$,

$$
\lim _{r \rightarrow \infty, r \notin E} \frac{N_{\kappa}^{f^{k}}\left(r, H_{j_{0}}\right)}{T\left(r, f^{k}\right)}=1 \quad(0 \leq k \leq M) .
$$

Proof. Since $N_{\kappa}^{f^{0}}\left(r, H_{j}\right)=\cdots=N_{\kappa}^{f^{M}}\left(r, H_{j}\right)$, Proposition 4.7 (iii) gives

$$
\begin{aligned}
\|(q-N & -1) T(r) \\
& \leq(M+1) \sum_{j=1}^{q} N_{\kappa}^{f^{k}}\left(r, H_{j}\right)+o(T(r)) \\
& \leq \frac{(M+1) \kappa}{M} T(r)+\left(1-\frac{\kappa}{M}\right) \sum_{k^{\prime}=0}^{M} N_{\kappa}^{f^{k^{\prime}}}\left(r, H_{j_{0}}\right)+o(T(r)) \\
& \leq(M+1) \frac{\kappa}{M} T(r)+\left(1-\frac{\kappa}{M}\right) T(r)+o(T(r)) \\
& \leq(\kappa+1) T(r)+o(T(r))
\end{aligned}
$$


for $k=0,1, \cdots, M$. By the same argument as in the proof of Corollary 4.8, we have $q \leq N+\kappa+2$.

Assume that $q=N+\kappa+2$. Then, by the above inequalities,

$\|(\kappa+1) T(r) \leq(M+1) \sum_{j=1}^{q} N_{\kappa}^{f^{k}}\left(r, H_{j}\right)+o(T(r)) \leq(\kappa+1) T(r)+o(T(r))$.

This concludes that, for a subset $E$ of $[0,+\infty)$ with $\int_{E} d r<+\infty$,

$$
\lim _{r \rightarrow \infty, r \notin E} \frac{T(r)}{\sum_{j=1}^{q} N_{\kappa}^{f^{k}}\left(r, H_{j}\right)}=\frac{M+1}{\kappa+1} .
$$

On the other hand, as is easily seen from Theorem 2.13 ,

$$
\limsup _{r \rightarrow \infty, r \notin E} \frac{T\left(r, f^{k}\right)}{\sum_{j=1}^{q} N_{\kappa}\left(r, H_{j}\right)} \leq \frac{1}{\kappa+1} .
$$

Therefore, for each $k$ we obtain

$$
\begin{aligned}
\frac{M+1}{\kappa+1} & \leq \liminf _{r \rightarrow \infty, r \notin E} \frac{T\left(r, f^{k}\right)}{\sum_{j=1}^{q} N_{\kappa}^{f^{k}}\left(r, H_{j}\right)}+\sum_{k^{\prime} \neq k} \limsup _{r \rightarrow \infty, r \notin E} \frac{T\left(r, f^{k^{\prime}}\right)}{\sum_{j=1}^{q} N_{\kappa}^{f^{\prime}}\left(r, H_{j}\right)} \\
& \leq \sum_{k^{\prime}=0}^{M} \limsup _{r \rightarrow \infty, r \notin E} \frac{T\left(r, f^{k^{\prime}}\right)}{\sum_{j=1}^{q} N_{\kappa}^{f^{\prime}}\left(r, H_{j}\right)} \leq \frac{M+1}{\kappa+1} .
\end{aligned}
$$

In view of (4.14), we can conclude (4.11) for each $k$. Therefore, for all $k$ and $\ell$,

$$
\begin{aligned}
\lim _{r \rightarrow \infty, r \notin E} & \frac{T\left(r, f^{k}\right)}{T\left(r, f^{\ell}\right)} \\
= & \lim _{r \rightarrow \infty, r \notin E} \frac{T\left(r, f^{k}\right)}{\sum_{j=1}^{q} N_{\kappa}^{f^{k}}\left(r, H_{j}\right)} \lim _{r \rightarrow \infty, r \notin E} \frac{\sum_{j=1}^{q} N_{\kappa}^{f^{\ell}}\left(r, H_{j}\right)}{T\left(r, f^{\ell}\right)}=1 .
\end{aligned}
$$

Proposition 4.7 (iii) gives also

$$
\|(\kappa+1) T(r) \leq \frac{(M+1) \kappa}{M} T(r)+(M+1)\left(1-\frac{\kappa}{M}\right) N_{\kappa}^{f^{k}}\left(r, H_{j_{0}}\right)+o(T(r)) .
$$

Under additional condition $M>\kappa$, we have

$$
\liminf _{r \rightarrow \infty, r \notin E} \frac{N_{\kappa}^{f^{k}}\left(r, H_{j_{0}}\right)}{T(r)} \geq \frac{\kappa+1-\frac{(M+1) \kappa}{M}}{(M+1)\left(1-\frac{\kappa}{M}\right)}=\frac{1}{M+1} .
$$


By (4.12), we conclude

$$
(1 \geq) \liminf _{r \rightarrow \infty, r \notin E} \frac{N_{\kappa}^{f^{k}}\left(r, H_{j_{0}}\right)}{T\left(r, f^{k}\right)} \geq(M+1) \liminf _{r \rightarrow \infty, r \notin E} \frac{N_{\kappa}^{f^{k}}\left(r, H_{j_{0}}\right)}{T(r)} \geq 1 .
$$

This completes the proof of Theorem 4.10.

We can prove also the following improvements of [9, Corollary 4.5]:

THEOREM 4.15. Assume that $q=N+2 \kappa+1$. Then, for each map $f \in \mathcal{F}_{\kappa}\left(H_{1}, \ldots, H_{q} ; g ; 1\right)$ with $f \neq g$, there exists a set $E$ with finite measure such that

$$
\lim _{r \rightarrow \infty, r \notin E} \frac{\sum_{j=1}^{q} N_{1}^{f}\left(r, H_{j}\right)}{T(r, f)}=2
$$

and

$$
\lim _{r \rightarrow \infty, r \notin E} \frac{T(r, f)}{T(r, g)}=1 .
$$

Proof. As in the proof of Corollary 4.8, we set $f^{0}:=f$ and $f^{1}:=g$. By the assumption, there is some $c \in \mathcal{C}$ and $j_{0}$ with $\Phi^{0}\left(F_{c}^{0 j_{0}}, F_{c}^{1 j_{0}}\right)=$ $F_{c}^{0 j_{0}}-F_{c}^{1 j_{0}} \not \equiv 0$. Then, we can apply Proposition 4.7 (ii) for the case $M=1$ and $\ell_{0}=1$ to get

$$
\| 2 \kappa T(r) \leq 2 \kappa \sum_{j=1}^{q} N_{1}^{f^{k}}\left(r, H_{j}\right)+o(T(r)) \leq 2 \kappa T(r)+o(T(r))
$$

for $k=0,1$. This gives

$$
\lim _{r \rightarrow \infty, r \notin E} \frac{T\left(r, f^{0}\right)+T\left(r, f^{1}\right)}{\sum_{j=1}^{q} N_{1}^{f^{k}}\left(r, H_{j}\right)}=1
$$

for a set $E$ with finite measure. On the other hand, Theorem 2.13 yields

$$
\limsup _{r \rightarrow \infty, r \notin E} \frac{T\left(r, f^{k}\right)}{\sum_{j=1}^{q} N_{1}^{f^{k}}\left(r, H_{j}\right)} \leq \limsup _{r \rightarrow \infty, r \notin E} \frac{\kappa T\left(r, f^{k}\right)}{\sum_{j=1}^{q} N_{\kappa}^{f^{k}}\left(r, H_{j}\right)} \leq \frac{1}{2},
$$

whence

$$
\lim _{r \rightarrow \infty, r \notin E} \frac{\sum_{j=1}^{q} N_{1}^{f^{0}}\left(r, H_{j}\right)}{T\left(r, f^{0}\right)}=\lim _{r \rightarrow \infty, r \notin E} \frac{\sum_{j=1}^{q} N_{1}^{f^{1}}\left(r, H_{j}\right)}{T\left(r, f^{1}\right)}=2
$$


and

$$
\begin{aligned}
\lim _{r \rightarrow \infty, r \notin E} & \frac{T\left(r, f^{0}\right)}{T\left(r, f^{1}\right)} \\
= & \lim _{r \rightarrow \infty, r \notin E} \frac{T\left(r, f^{0}\right)}{\sum_{j=1}^{q} N_{1}^{f^{0}}\left(r, H_{j}\right)} \lim _{r \rightarrow \infty, r \notin E} \frac{\sum_{j=1}^{q} N_{1}^{f^{1}}\left(r, H_{j}\right)}{T\left(r, f^{1}\right)}=1 .
\end{aligned}
$$

For three meromorphic maps, we have the following:

THEOREM 4.17. In the situation stated at the beginning of this section, consider the case $M=2$ and $\ell_{0}=2$ and assume that $\Phi^{\alpha}:=\Phi^{\alpha}\left(F_{c}^{0 j_{0}}, F_{c}^{1 j_{0}}\right.$, $\left.F_{c}^{2 j_{0}}\right) \not \equiv 0$ for some $c \in \mathcal{C}, j_{0}$ and $\alpha$ with $|\alpha|=1$. If $q=N+2 \kappa+1$, then

$$
\lim _{r \rightarrow \infty, r \notin E} \frac{N_{1}^{f^{k}}\left(r, H_{j_{0}}\right)}{T\left(r, f^{k}\right)}=1 \quad \text { for } k=0,1,2 .
$$

Proof. We apply Proposition 4.7 (ii) for the case $M=2, \ell_{0}=2$ and $q=N+2 \kappa+1$. Then, we have

$$
\| 2 \kappa T(r) \leq \frac{3 \kappa}{2} T(r)+\frac{\kappa}{2} \sum_{k=0}^{2} N_{1}^{f^{k}}\left(r, H_{j_{0}}\right)+o(T(r)),
$$

whence

$$
\liminf _{r \rightarrow \infty, \notin E} \frac{\sum_{k=0}^{2} N_{1}^{f^{k}}\left(r, H_{j_{0}}\right)}{T(r)} \geq 1
$$

By the help of (4.16), we have the desired conclusion.

\section{$\S 5$. Degeneracy theorems for some sets of meromorphic maps}

As in the previous section, taking a $\kappa$-nondegenerate meromorphic map $g$, a positive integer $\ell_{0}$ and hyperplanes $H_{1}, \ldots, H_{q}$ in general position with $\operatorname{dim} \bigcup_{i<j} g^{-1}\left(H_{i} \cap H_{j}\right) \leq n-2$, we consider the set $\mathcal{F}_{\kappa}\left(H_{1}, \ldots, H_{q} ; g ; \ell_{0}\right)$.

We now give the following theorem, which yields Theorem 1.3 as $\kappa=N$.

THEOREM 5.1. Suppose that $q \geq N+\kappa+2$ and $\ell_{0}=\kappa(\kappa+1) / 2+\kappa$, and take arbitrary maps $f^{0}, \ldots, f^{\kappa+1}$ in $\mathcal{F}_{\kappa}\left(H_{1}, \ldots, H_{q} ; g ; \ell_{0}\right)$. Then, there are $N+1$ hyperplanes $H_{j_{0}}, H_{j_{1}}, \ldots, H_{j_{N}}$ among $H_{j}$ 's such that, if we choose homogeneous coordinates $\left(w_{0}: \cdots: w_{N}\right)$ on $P^{N}(\mathbb{C})$ with $H_{j_{i}}=\left\{w_{i}=0\right\}$ $(0 \leq i \leq N)$ and take reduced representations $f^{k}=\left(f_{0}^{k}: \cdots: f_{N}^{k}\right)$, then

$$
\frac{f_{i}^{1}}{f_{j}^{1}}-\frac{f_{i}^{0}}{f_{j}^{0}}, \frac{f_{i}^{2}}{f_{j}^{2}}-\frac{f_{i}^{0}}{f_{j}^{0}}, \ldots, \frac{f_{i}^{\kappa+1}}{f_{j}^{\kappa+1}}-\frac{f_{i}^{0}}{f_{j}^{0}}
$$

are linearly dependent over $\mathbb{C}$ for each pair $(i, j)$ with $0 \leq i, j \leq N$. 
Proof. We set $M:=\kappa+1$ and use the same notation as in the previous section. Consider the subset

(5.2) $J:=\left\{j ; \psi_{c}^{1 j}, \ldots, \psi_{c}^{M j}\right.$ are linearly dependent for every $\left.c \in \mathcal{C}\right\}$.

of $\{1,2, \ldots, q\}$. We take a subset $E$ of $[0,+\infty)$ with finite measure such that Theorems 2.6 and 2.13 hold for all meromorphic maps $f^{k}$. By (4.13), if $j_{0} \notin J$, then $\liminf _{r \rightarrow \infty, r \notin E} N_{1}\left(r, H_{j_{0}}\right) / T\left(r, f^{k}\right)=1$ and, by (4.11), the number of indices not contained in $J$ is at most $\kappa+1$. Therefore, $J$ contains at least $N+1$ indices. Let $\left\{j_{0}, j_{1}, \ldots, j_{N}\right\}$ and we choose homogeneous coordinates $\left(w_{0}: \cdots: w_{N}\right)$ on $P^{N}(\mathbb{C})$ so that $H_{j_{i}}=\left\{w_{i}=0\right\}(0 \leq i \leq N)$. Then, for each $j \in J$ and $c \in \mathcal{C}$,

$$
\Phi^{\alpha}\left(F_{c}^{0 j}, \ldots, F_{c}^{M j}\right) \equiv 0 \quad \text { for all } \alpha \text { with }|\alpha| \leq M(M+1) / 2 .
$$

Since $\mathcal{C}$ is dense in $\left(\mathbb{C}^{N+1}\right)_{*}$ by Lemma 4.4 and $\Phi^{\alpha}\left(F_{c}^{0 j}, \ldots, F_{c}^{M j}\right)$ depends continuously on parameters $c,(5.3)$ holds for every $c \in\left(\mathbb{C}^{n+1}\right)_{*}$, particularly, for $c_{j}:=(0, \ldots, 0, \stackrel{j \text {-th }}{1}, 0, \ldots, 0)$. By Proposition 3.3 we can easily conclude that $f_{i}^{1} / f_{j}^{1}-f_{i}^{0} / f_{j}^{0}, \ldots, f_{i}^{\kappa+1} / f_{j}^{\kappa+1}-f_{i}^{0} / f_{j}^{0}$ are linearly dependent over $\mathbb{C}$ for $0 \leq i, j \leq N$.

We next study the case $\ell_{0}=2$.

THEOREM 5.4. Suppose that $q \geq N+2 \kappa+1$ and take three maps $f^{0}$, $f^{1}, f^{2}$ in $\mathcal{F}_{\kappa}\left(H_{1}, \ldots, H_{q} ; g ; 2\right)$ with reduced representations $f^{k}=\left(f_{0}^{k}: \cdots\right.$ : $\left.f_{N}^{k}\right)$. Set

$$
\chi_{f^{k}}^{i j}:=\frac{a_{i 0} f_{0}^{k}+\cdots+a_{i N} f_{N}^{k}}{a_{j 0} f_{0}^{k}+\cdots+a_{j N} f_{N}^{k}} .
$$

Then, after a suitable change of indices, for each $i, j$ with $1 \leq i, j \leq$ $N+2 \kappa-1$ there exist some nonzero constatnts $c_{i j}$ such that

$$
\chi_{f^{0}}^{i j}=c_{i j} \chi_{f^{1}}^{i j}, \chi_{f^{1}}^{i j}=c_{i j} \chi_{f^{2}}^{i j} \text { or } \chi_{f^{2}}^{i j}=c_{i j} \chi_{f^{0}}^{i j}
$$

where $c_{i j}=1$ for each $i, j$ in the case $\kappa \geq 2$.

For the proof, we first give the following lemma.

LEMMA 5.5. Under the same assumption as in Theorem 5.4, if $\kappa>1$ and $\chi_{f^{0}}^{i j} / \chi_{f^{1}}^{i j}$ is a constant for some $i, j$, then $\chi_{f^{0}}^{i j}=\chi_{f^{1}}^{i j}$. 
Proof. By the assumption, there is a nonzero constant $C$ with $\chi_{f^{1}}^{i j}=$ $C \chi_{f^{0}}^{i j}$, where $\chi_{f^{0}}^{i j} \neq 0$ because $f^{0}$ is nondegenerate. Assume that $C \neq$ 1. By the assumption $f^{0}=f^{1}$ on $\bigcup_{j=1}^{q}\left(f^{0}\right)^{-1}\left(H_{j}\right)$, we have $\chi_{f^{0}}^{i j}=0$ on $\bigcup_{j^{\prime} \neq j}\left(f^{0}\right)^{-1}\left(H_{j^{\prime}}\right)$. It follows that

$$
\begin{aligned}
\|(2 \kappa-1) T\left(r, f^{0}\right) & \leq \sum_{j^{\prime} \neq j} N_{\kappa}^{f^{0}}\left(r, H_{j^{\prime}}\right)+o\left(T\left(r, f^{0}\right)\right) \\
& \leq \kappa \sum_{j^{\prime} \neq j} N_{1}^{f^{0}}\left(r, H_{j^{\prime}}\right)+o\left(T\left(r, f^{0}\right)\right) \\
& \leq \kappa N\left(r, \nu_{\chi_{f^{0}}^{0}}{ }^{i j}\right)+o\left(T\left(r, f^{0}\right)\right) \\
& \leq \kappa T\left(r, f^{0}\right)+o\left(T\left(r, f^{0}\right)\right)
\end{aligned}
$$

We then have $\kappa \leq 1$, which contradicts the assumption. This concludes $C=1$.

Proof of Theorem 5.4. As in the proof of Theorem 5.1, we consider the set $J$ defined by (5.2). In this case, by Theorems 4.15 and $4.17 J$ contains at least $N+2 \kappa-1$. After a suitable change of indices, we may assume $\{1,2, \ldots, N+2 \kappa-1\} \subseteq J$. By the same reason as in the proof of Theorem 5.1 , there are some nonzero vectors $\left(\lambda_{i j}^{0}, \lambda_{i j}^{1}, \lambda_{i j}^{2}\right)$ such that

$$
\lambda_{i j}^{0}+\lambda_{i j}^{1}+\lambda_{i j}^{2}=0, \lambda_{i j}^{0} \chi_{f^{0}}^{i j}+\lambda_{i j}^{1} \chi_{f^{1}}^{i j}+\lambda_{i j}^{2} \chi_{f^{2}}^{i j}=0
$$

Since $\chi_{f^{k}}^{j i}=1 / \chi_{f^{k}}^{i j}$, we can apply Proposition 3.4 to show that there are some nonzero constants $c_{i j}(1 \leq i, j \leq N+2 \kappa-1)$ such that

$$
\chi_{f^{0}}^{i j}=c_{i j} \chi_{f^{1}}^{i j}, \chi_{f^{1}}^{i j}=c_{i j} \chi_{f^{2}}^{i j} \text { or } \chi_{f^{2}}^{i j}=c_{i j} \chi_{f^{0}}^{i j} .
$$

Here, by Lemma $5.5 c_{i j}=1$ for each $i, j$ if $\kappa>1$. These conclude Theorem 5.4.

Proof of Theorem 1.4. Assume that there exist three distinct maps $f^{0}$, $f^{1}, f^{2} \in \mathcal{F}\left(H_{1}, \ldots, H_{q} ; g ; 2\right)$, where we may take $\kappa=N$. First, we consider the case $N=1$ and so $q=4$ in Theorem 1.4. After a suitable change of indices, $\chi_{f^{0}}^{12} / \chi_{f^{1}}^{12}, \chi_{f^{1}}^{12} / \chi_{f^{2}}^{12}$ and $\chi_{f^{2}}^{12} / \chi_{f^{0}}^{12}$ are all constants. Then, there is a Möbius transformation $w^{\prime}=L(w)$ with $f^{1}=L\left(f^{0}\right)$. In this situation, since the number of fixed values for $L(w)$ are at most two, each $f^{k}$ omits two of 
$H_{j}$ 's, and $L(w)$ interchanges them with each other and fixes others, whence $L(w)$ is unique. Since the same conclusion holds for the maps $f^{2}$ and $f^{0}$. These give an absurd conclusion $f^{1}=f^{2}=L\left(f^{0}\right)$.

For the case $N \geq 2$, by Lemma 5.5, $c_{i j}=1$ for all constants $c_{i j}$ mentioned in Theorem 5.4. Therefore, either $\chi_{f^{0}}^{i j}=\chi_{f^{1}}^{i j}, \chi_{f^{1}}^{i j}=\chi_{f^{2}}^{i j}$ or $\chi_{f^{2}}^{i j}=\chi_{f^{0}}^{i j}$ holds for each $i$ and $j$ with $1 \leq i, j \leq 3 N-1$. Particularly, for $L:=3 N-1$, $\chi_{f^{0}}^{j L}=\chi_{f^{1}}^{j L}, \chi_{f^{1}}^{j L}=\chi_{f^{2}}^{j L}$ or $\chi_{f^{2}}^{j L}=\chi_{f^{0}}^{j L}$ for $1 \leq j \leq 3 N-2$. Consider the subset

$$
I_{1}:=\left\{j ; \chi_{f^{0}}^{j L}=\chi_{f^{1}}^{j L}\right\}, I_{2}:=\left\{j ; \chi_{f^{1}}^{j L}=\chi_{f^{2}}^{j L}\right\}, I_{3}:=\left\{j ; \chi_{f^{2}}^{j L}=\chi_{f^{0}}^{j L}\right\}
$$

of $\{1,2, \ldots, 3 N-2\}$. One of these contains at least $N$ indices. In fact, if all of $I_{1}, I_{2}$ and $I_{3}$ contain at most $N-1$ indices, then we have an absurd conclusion

$$
3 N-2 \leq \#\left(I_{1} \cup I_{2} \cup I_{3}\right) \leq \# I_{1}+\# I_{2}+\# I_{3} \leq 3(N-1) .
$$

This gives the desired conclusion $f^{0}=f^{1}, f^{1}=f^{2}$ or $f^{2}=f^{0}$.

\section{REFERENCES}

[1] H. Cartan, Un nouveau théorème d'unicité relatif aux fonctions méromorphes, C. R. Acad. Sci. Paris, 188 (1929), 301-30.

[2] H. Fujimoto, The uniqueness problem of meromorphic maps into the complex projective space, Nagoya Math. J., 58 (1975), 1-23.

[3] H. Fujimoto, A uniqueness theorem of algebraically non-degenerate meromorphic maps into $P^{N}(\mathbb{C})$, Nagoya Math. J., 64 (1976), 117-147.

[4] H. Fujimoto, Remarks to the uniqueness problem of meromorphic maps into $P^{N}(\mathbb{C})$, I, Nagoya Math. J., 71 (1978), 13-24.

[5] H. Fujimoto, Non-integrated defect relation for meromorphic maps of complete Kähler manifolds into $P^{N_{1}}(\mathbb{C}) \times \cdots \times P^{N_{k}}(\mathbb{C})$, Japanese J. Math., 11 (1985), 233-264.

[6] H. Fujimoto, A unicity theorem for meromorphic maps of a complete Kähler manifold into $P^{N}(\mathbb{C})$, Tôhoku Math. J., 38 (1986), 327-341.

[7] H. Fujimoto, Unicity theorems for the Gauss maps of complete minimal surfaces, J. Math. Soc. Japan, 45 (1993), 481-487.

[8] H. Fujimoto, Unicity theorems for the Gauss maps of complete minimal surfaces, II, Kodai Math. J., 16 (1993), 335-354.

[9] S. Ji, Uniqueness Problem without multiplicities in value distribution theory, Pacific J. Math., 135 (1988), 323-348.

[10] R. Nevanlinna, Einige Eindeutigkeitssätze in der Theorie der meromorphen Funktionen, Acta Math., 48 (1926), 367-391. 
[11] B. Shiffman, Introduction to the Carlson-Griffiths equidistribution theory, Lecture Notes in Math., 981, Springer-Verlag, 1983.

[12] L. Smiley, Geometric conditions for unicity of holomorphic curves, Contemporary Math., 25 (1983), pp. 149-154.

[13] N. Steinmetz, A uniqueness theorem for three meromorphic functions, Annales Acad. Sci. Fenn., 13 (1988), 93-110.

Department of Mathematics

Faculty of Science

Kanazawa University

Kakuma-machi, Kanazawa, 920-11

Japan

fujimoto@kappa.s.kanazawa-u.ac.jp 